Hughes, W. H. (1957). J. gen. Microbiol. 17, 49-58

\title{
A Reconsideration of the Swarming of Proteus vulgaris
}

\author{
BY W. HOWARD HUGHES
}

The Wright-Fleming Institute, St Mary's Hospital, London, W. 2

SUMMARY: Studies by cinemicrography confirm that the long forms of Proteus vulgaris (Hauser) seen during the swarming stage are morphologically indistinguishable from those induced by penicillin and other toxic agents. They appear only in cultures which are growing freely and when a threshold concentration of population has been reached; thereafter the organisms at the growing edge of the colony become more abnormal with each swarming. The normal small bacilli may begin to move before any long forms are present. In later swarmings, movement may be seen in small as well as in long organisms. It is suggested that the long forms are induced by a non-specific volatile agent which can not be detected when growth has been cleared from the medium. Its action is enhanced in cultures which are confined under a coverslip. Though it is partially neutralized by the action of catalase, conclusive proof of its nature is still lacking. When different strains are grown together long forms are soon produced. The agents causing this are specific and stable, producing their effect immediately, and are detectable in the medium after the primary culture has been cleared from it.

The swarming of Proteus vulgaris (Hauser) has received little attention recently. Since the monograph of Moltke (1927) few papers containing new observations have appeared. Mayr-Harting (Russ-Munzer, 1935) and Lominski \& Lendrum (1947) offered different interpretations of the phenomenon. The interference of incompatible strains was studied by Dienes $(1946,1947)$ and by Cunliffe \& Krickler (1953), who together with Kvittingen (1949, 1953) and Klieneberger-Nobel (1947), are among the few who have added anything to the earlier results.

During studies on the action of various damaging agents on bacteria (Hughes, 1956) it became clear that the spontaneous changes among swarming cells were like those caused by some chemical and the other traumata. Further, the mortality among these cells was exceedingly high, as Moltke (1927) had said. Since the earlier workers had reported their results, new techniques, notably timelapse cinemicrography and the use of the micromanipulator, have made the prolonged study of individual cells possible. It was decided to apply these techniques to study the nature of the phenomenon, and if possible to bring it into line with other examples of abnormal cell morphology.

\section{METHODS}

Technique 1. Buffered nutrient agar, 2-3\% was filtered through an F.C.B. Sterimet in a steamer to remove the debris and dead bacteria. These, though normally unimportant, may be mistaken for part of the implant of living cells when photographed. Before cooling the $\mathrm{pH}$ value was again adjusted to diminish precipitation of phosphates since these confuse the background. When required for use, the medium was melted and poured into heated Petri dishes, 
reasonably free from flaws, to give a uniform layer not more than $2 \mathrm{~mm}$. thick. After cooling slowly at room temperature the medium was dried only long enough to remove surface moisture. The plates were inoculated with a straight wire as near the centre as possible.

At intervals sectors were cut, so that the whole of the growth from the site of inoculation to the colony edge, together with a clear area of unoccupied agar, was represented in the same relative amount as on the original plate. The extreme edge was removed with a scalpel, as its inclusion gives an optically unsatisfactory wedge and, when a coverslip is applied, the pressure is uneven and expressed fluid will collect. Both covered and uncovered preparations were examined at $37^{\circ}$. For prolonged observation the coverslips were sealed with paraffin wax. The plate from which the sector had been taken was also incubated as a control for any action due to the coverslip or to the separation of organisms from the colony.

Technique 2. While a coverslip resting on the agar was adequate for photography at normal speeds, it had disadvantages in timelapse work since the weight of the coverslip compressed the agar slowly and progressively and the field fell out of focus. This was overcome by making slide cells from dark gound slides. Two slats of glass $0.5 \mathrm{~cm}$. wide were cut from a slide and cemented on to an intact slide $1.0 \mathrm{~cm}$. apart. At the suggestion of Dr B. A. D. Stocker they were secured by Picein wax (W. Edwards and Son). Coverslips, $1.5 \mathrm{~cm}$. wide, were also cut from slides. The organisms to be studied were dried on the coverslip, melted agar was poured between the two supports and the slip inverted on to this, pressed down and sealed with paraffin wax.

Technique 3. Where records of the whole area of the plate had to be made by timelapse the camera's own lens system was used.

Indirect oblique lighting from a $1000 \mathrm{~W}$. studio lamp with a reflector was thrown by a mirror on to the Petri dish; this dish was supported on a sheet of glass and its optical defects rendered inconspicuous by running glycerol between the two surfaces in contact. A black velvet cloth was spread some distance below the glass. To prevent drying and to lift the cover of the dish out of the focal plane of the camera, a cylinder of Perspex of the same diameter as the dish and $8 \mathrm{in}$. high was seecurd to it by transparent tape. The upper end of the cylinder was closed with a sheet of plate glass and masked with a circle of black paper. To control condensation on this cover a bowl type $\frac{1}{2} \mathrm{~kW}$. electric fire was focused on it. Screens were suspended to protect the camera and other parts of the apparatus from direct light and heat.

Strains. The strains of Proteus vulgaris used in this work were 'P. 26', 'Utton' and 'Maye'. All had been repeatedly reselected with a micromanipulator and grown from single organisms. All these strains were of different types and on the same plate would not swarm into one another.

\section{Examination of material and recording of results}

The mounted sectors were examined by phase contrast, the microscope being enclosed in a Perspex incubator. The stages of growth were recorded by timelapse photography at a rate of one frame every 18 sec. When movement 
began, whether this was typical swarming, flagellar movement or the movement of normal cells, it was photographed at normal speed. Between exposures direct observation was maintained until further change was noticed, when this also was recorded. When the colony, by swarming, had covered a considerable area, a series of exposures were made from the edge to the site of the original inoculation.

After swarming the fate of the individual microbes was again followed by timelapse photography. Several different arrangements were made; $(a)$ the preparation was left entire, covered with a slip and sealed; $(b)$ it was left uncovered and followed until drying interfered with observation; $(c)$ the swarming edge was cut from the rest of the colony and isolated; $(d)$ swarm cells were transferred to fresh medium. Comparable sectors from the control plates were also examined.

\section{Primary magnifications}

Since the distance from the eyepiece to the camera gives a $\times 3$ enlargement, to obtain a reasonable field it is necessary to decrease the magnification of the microscope. In general a $\times 20$ objective with $a \times 6$ or $\times 8$ eyepiece was enough. The resolution was not high and for finer detail the $\times 95$ oil-immersion lens with the Baker $\times 1 \frac{1}{2}$ eyepiece was more suitable.

\section{Photographic details}

After preliminary work with Kodak cinemicrofilm it was found that Kodak Super $\mathbf{X}$ reversal film was the most satisfactory as it allowed both timelapse photography, with a $\times 36$ mercury green filter, and normal speed work, with a $\times 3$ yellow-green filter.

Exposures of $\frac{1}{2}$ sec. were given since the shutter of most cinecameras give inconstant results on the first frame and this is smoothed over with the longer time exposure.

\section{RESULTS}

On solid medium growth begins normally and nothing distinguishes the Proteus cells from those of other bacillary organisms. This phase usually lasts for about $3 \mathrm{hr}$. at $37^{\circ}$ and varies with the strain and the concentration of agar in the medium or the amount of drying.

When the colony has reached a diameter of about $8 \mathrm{~mm}$. swarming begins. There will be a raised edge to the colony, and though no sign of swarming will be visible to the naked eye, continuous microscopic observation detects the first signs of activity. No long forms are then present but a small number of organisms become motile around the perimeter. This phase is transient and only prominent where islands of unoccupied medium have been left by the uneven spreading growth of the colony. Round these islands the movement is taken up by a complete line of organisms and continues until the space has been filled by them.

A further period of growth without visible independent movement of any of the organisms lasts for half an hour before the second phase of movement 
begins. The organisms separate out into two zones, the longer on the outside and the more motile, shorter ones between them and the main mass forming the old colony edge. The movement continues until the space has filled; these cells come in from the edge of the old colony, but during this time division is presumably also taking place: During the resting period which follows, small and large cells divide in the normal way without evidence that they are inhibited. The movements of small cells described above in covered preparations is also seen in uncovered ones.

The same sequence is repeated after another interval, but is now more obvious and there are three, rather than two, zones; movement can be detected under a hand-lens since there are now small irregularities projecting from the colony. Next to the solid colony is a palisade of long cells, then a space full of many active small cells and finally beyond this a further palisade. From both lines of long cells individuals swim out, their places being taken by others which have been swimming between the two palisades. After each of these cycles of activity, and as a result of the pattern left by the palisading, three rings are visible at the colony edge, easily misinterpreted as the result of three growth cycles instead of only one.

The fourth phase of activity is obvious to the naked eye and is the typical swarming reported by all previous workers. Groups of long forms push out over the medium, some are motile while others are inert and are simply carried along, being deposited at a distance from the colony edge and not infrequently picked up again by other passing groups. There is no evidence of negative chemotaxis since the groups return to the main mass when deflected, and at the peak of activity movement is random.

The maximum movement is not at the edge of the colony but some distance in. Here the violently active cells can be seen to divide while still motile and form trains of cells which follow each other through the lines of least resistance in the mass. At the site of the original implant only small forms are present; these show active movement though this is hampered by their numbers. Activity ends gradually, first in the small cells in the centre, then among the swarmers and last among the small forms derived from swarmers some distance from the edge. Subculture or continuous observation of long forms still undivided at the end of swarming shows that most of them are not viable, as was shown in the following experiments:

\section{Experiment 1}

The disk of agar carrying the growth up to the point where swarming began was cut from a colony, leaving a ring carrying mainly swarmers. Blocks were cut from this and mounted under coverslips and the fate of the swarmers recorded by timelapse photography.

Result. During the period up to $72 \mathrm{hr}$. no cell division was seen in covered preparations; the organisms became progressively less well defined and the contrast between them and the surrounding medium diminished. 


\section{Experiment 2}

The organisms were transferred to fresh medium under a coverslip. They were examined visually at intervals of $30 \mathrm{~min}$. by phase contrast and those giving rise to micro-colonies were counted.

Result. This experiment has been previously reported in detail (Hughes, $1953 b$ ). The number giving rise to normal small forms decreases after each successive swarming, being as high as $\mathbf{5 0} \%$ with the first visible swarms, and falling to a fraction of $1 \%$ in fourth and subsequent swarms.

The suppression of swarming by altering the concentration of the agar in the medium does not prevent the production of abnormal forms; although these strains are unable to progress on concentrated agar above $3 \%$ the beating of their flagella can be detected under oil-immersion and, when the medium is moistened, they move freely on it. Drying of the medium during prolonged incubation is probably responsible for the irregularity of the later swarms mentioned by Russ-Münzer (1935). Sectoring may occur with the production of variants which are more tolerant of higher concentrations (Pl. 1, fig. 1). Unilateral drying demonstrates the effect of concentration particularly well (Pl. 1, fig. 2).

\section{The nature of the toxic effect}

The interaction of Proteus strains reported by Dienes (1946, 1947), and studied by Cunliffe \& Krickler (1953), indicates there is some toxic effect when one strain acts on another. These workers showed that when two cultures of the same strain were planted on a plate together, when the swarms met they would join up and spread together. When different strains are on the same plate a line of separation is left between the swarms and the organisms on either side of the line are particularly bizarre in appearance. It has not previously been reported, however, that a similar action can be detected microscopically when strains are mixed and seeded together on solid medium, long forms then being produced after only a few divisions. This interaction cannot account for the development of swarmers from a pure strain since only compatible organisms are present. Again, it is well known that often when a broth culture is transferred to solid media some of the organisms may not divide and so become long forms. This is particularly well known with coliform organisms and with the salmonellas (Hughes, 1953a). These long organisms lyse early and are eliminated from the cultures, while the shorter bacilli establish themselves on artificial medium. This is entirely different from the Proteus long cell which only appears when colonial growth has reached an advanced stage.

While investigating this, most of Moltke's experiments were repeated with minor modifications and his results essentially confirmed. Only one or two need mention as of particular interest. He showed, for example, that when the agar on which a strain had grown was cleared of growth and remelted with added nutrients, or turned over and planted on the back, it was as acceptable to the strain as it was originally and no premature swarming occurred. 
The experiment is simpler to perform when the organism is grown on a cellophan or collodion membrane; it is then unnecessary to remelt the agar, and clearing is more complete.

\section{Experiment 3}

A collodion membrane, average pore size $0.89 \mu$, diameter $4 \mathrm{~cm}$. was placed in the middle of a nutrient agar plate. A drop of broth suspension was placed near the centre and the preparation incubated at $37^{\circ}$. Growth and swarming took place in the usual way.

The membrane with the growth was then removed leaving a ring of swarming cells beyond the edge. The centrifugal spread of the colony continued, but no swarming back on to the bare area took place.

This at first sight appears to be similar to the experiments of Lominski \& Lendrum (1947), in which they showed that an area in the centre of a plate surrounded with growth is frequently not invaded but remains clear even when the whole of the rest of the plate has been occupied. This observation I have confirmed. It supports the idea of a negatively chemotaxic and, possibly, toxic agent, but it is important to note a difference between these authors' experiments and my own. The bare area in their cultures was essentially normal medium and was eventually occupied by a thin film of growth. The cleared one which had been under a membrane was depleted of nutrients and corresponded to that used by Moltke.

\section{Experiment 4}

The preparation was made as before but the membrane was removed with the entire culture before the swarms had reached the edge. The cleared plate was re-inoculated with a streak of organisms near to one side. Swarming across the plate was at first typical, but the site of the previous growth was avoided, leaving a bare area similar in shape.

\section{Experiment 5}

Blocks of agar were cut from this cleared area, mounted on slides and inoculated with cultures of the original strain P. 26, and incompatible strains 'Maye' and 'Utton'.

Result. Re-inoculation of the cleared area with a fresh inoculum of the same strain was followed by only a slow growth of cells of normal morphology. When, however, cells of an incompatible strain were used, long forms appeared as soon as if the strains had been grown in a mixture. Provisionally, then, we may assume that there are at least two toxic agents at work; one is retained in the medium, is relatively stable and the product of strains detected only in the presence of another, while a second is active only in crowded cultures and not specific in any way.

Peroxide accumulation would explain what we know of this latter agent. It might accumulate in areas of dense growth and probably disappear quickly from remelted agar or after clearing of the growth. It would not reappear 
unless growth again passed a certain threshold. Further, when a block of agar is cut from a plate with the swarmers on it and mounted under a coverslip, virtually all the cells will degenerate; but in a comparable area of the original growth, treated in the same way but uncovered, such extensive changes are not seen. That the action is in fact due to peroxide is not demonstrable. Proteus still swarms on catalase-rich medium, for example on blood-agar.

The action of catalase was shown in the following way:

\section{Experiment 6}

A plate of nutrient agar was divided into two halves by a ditch. One half was treated with a $1 \%$ solution of catalase (containing $10 \%$ of the active enzyme), the other was left as a control. At one end of the plate organisms were streaked at right angles to the ditch.

Result. The zones of swarming appeared sooner on the untreated side, being visible to the naked eye in $8 \mathrm{hr}$., while in the presence of catalase nearly $18 \mathrm{hr}$. elapsed. The width of the swarms was $0.8 \mathrm{~cm}$. on the untreated side and $0 \cdot 1-0 \cdot 2 \mathrm{~cm}$. on the treated.

When samples of swarmers were transferred to catalase agar, even under a coverslip, they recovered and divided normally as short cells, while the control cells under a coverslip lysed, or, if uncovered, remained abnormal. This is inconclusive since a similar but less marked result was obtained on any fresh medium.

Since it is held that under anaerobic conditions peroxide may not be formed, a further series of investigations were done on anaerobic cultures. If in a McIntosh and Fildes's jar plates were maintained in a humid atmosphere, the growth of Proteus rapidly spread without any zoning being seen and it was impossible to distinguish any effect on the cultures: dry anaerobic cultures were made in the following way:

\section{Experiment 7}

An anaerobic jar was prepared with a dish of phosphorus pentoxide in it and the jar evacuated an hour before hydrogen was introduced. Control plates were placed in identical jars but these were not evacuated. The jars were incubated either at room temperature or at $37^{\circ}$.

Result. The dry anaerobic plates showed the same zoning as the aerobic controls, and similar stages of spreading and of morphological change were reached in the same time. Finally the effect of the direct addition of hydrogen peroxide to the culture was tried.

\section{Experiment 8}

Peroxide was introduced into a slide cell in which Proteus vulgaris was growing on agar, in the hope that it would diffuse into the medium and that organisms near it would be obviously affected while those at a distance would remain normal.

Result. No changes in morphology were seen. 


\section{Experiment 9}

Diffusion plates were prepared with ditches and punch holes in them. These were filled with peroxide. The plates were seeded with Proteus vulgaris and after various periods of incubation blocks were cut from them and the growth examined. The results were again negative. Finally one result was obtained suggesting that peroxide was itself playing some part.

\section{Experiment 10}

Into a row of 3 in. $\times \frac{1}{2}$ in. test tubes was placed $0.5 \mathrm{ml}$. broth. Serial dilutions of hydrogen peroxide at a nominal strength of 20 vol. were prepared in the usual way. To each tube was added one drop of an $18 \mathrm{hr}$. broth culture of Proteus vulgaris. A control without peroxide was also provided.

The preparations were incubated at $37^{\circ}$ and examined after $1,2,4$ and $17 \mathrm{hr}$. for growth and alteration of morphology. A second series was similarly prepared but the surface of the broth was covered with a layer of sterile liquid paraffin.

Result. After 2 and $4 \mathrm{hr}$. incubation the organisms in all concentrations above the equivalent of $0.6 \mathrm{vol}$. peroxide were non-motile. At $0.6 \mathrm{vol}$. long cells were seen. After $17 \mathrm{hr}$., growth was present up to $0.6 \mathrm{vol}$. and at this level only, organisms up to 7 times the normal maximum length for the strain were present. At no stage were any bulbs seen. Prolonged incubation did not bring about any further changes. The preparations sealed with paraffin were not more affected than the other series. The end-point for inhibition was also the same.

\section{DISCUSSION}

A number of apparently new observations justify this reconsideration of swarming. In the early stages no differences between Proteus and other bacillary forms could be seen. This is not in agreement with the views of Klieneberger-Nobel (1947) since she believed that there were differences between Proteus and coliform organisms at this early stage.

The early movement of small organisms need not be related to the formation of swarmers but it is not an artefact, due to the pressure of the coverslip on the raised edge squeezing out fluid from the medium, as Dr Mayr-Harting suggested (personal communication), and as I thought at first, since it is also regularly present in uncovered preparations. Further, the motility of the small organisms in the centre of the colony, difficult to study before phase contrast was discovered, suggests that there is a tendency to spontaneous movement by all the organisms and that this also may be independent of the formation of the swarmers though perhaps coincidental with it, both small and long forms being affected by the same stimulus to motility.

If other flagellated organisms, such as coliforms, are watched by timelapses cinemicrography from the stage of single cells to a colony, it may be some time before any cells are seen to be motile and after the first cells have swum away a compact colony may still be left. There is a suggestion here that 
some time may pass before cells which are potentially motile reveal this characteristic on solid or semi-solid medium. The time at which this motility will be seen varies greatly from species to species, and even between strains of the same species and clones within the strain. It is, of course, well known that long forms are as easily produced in non-motile organisms as in motile ones. Thus motility and the production of the typical long forms hitherto regarded as swarm cells need not be related.

If it be true that there is some toxic agent responsible for the transformation of normal organisms into long forms then its effect should be greatest when there is maximum concentration, which would be in the middle of the mass of growth, yet the swarmers occur almost exclusively at the edge of the colony. Comparison with the penicillin-induced long forms offers an explanation. If the culture is seeded on a high concentration of penicillin + nutrient agar, long forms will develop immediately, while in lower concentrations some apparently normal divisions will take place first. On a medium which will not support growth no long forms are produced. Bigger showed (1944) that the resting cell was relatively resistant. In the same way in cultures of Proteus vulgaris the consumption of nutrients at the centre of the colony will encourage a resting stage there and the cells will neither develop abnormally nor degenerate. At the edge, although the concentration of toxic substances may be less, growth will be taking place and so both abnormal morphology and degenerative changes will be seen.

Just as the motility and the development of swarmers may be separate processes, so also there may be several toxic agencies at work. Interference between strains is not the same phenomenon as auto-intoxication. The interstrain inhibitor remains in the medium after the original growth has been cleared from it, since re-inoculation with the same strain is followed by normal growth while with an heterologous strain growth is of long forms. One must postulate a group of strain-specific substances.

The general intoxication seen at the time of swarming is due to a more volatile agent. The two arguments for this are that (1) it cannot be detected in cleared medium, as Lominski \& Lendrum (1947) and Moltke (1927) also found, and (2) it is far more potent in a culture under a coverslip than in one exposed to the air. This last observation harmonizes various statements on the viability of swarmers. Direct proof that the substance is hydrogen peroxide is not provided either by the addition of peroxide to the medium or by transfer to catalase medium. The former fails to produce the more advanced morphological changes although there are some long forms and the latter, while it delays the appearance both of long forms and of swarming does not inhibit them.

It is fair to conclude that there are several chemical agents concerned in the swarming of Proteus and although the nature of these is not known the long forms are induced in essentially the same way as by penicillin. 
My thanks are due to my colleague, Dr Peter N. Cardew, head of the Photographic Department, for the supervision of all photographic techniques including the editing of the films.

Representative film sequences illustrating the results are available as a short sound film and may be obtained from the Photographic Department of St Mary's Hospital.

\section{REFERENCES}

Bigger, J. W. (1944). Treatment of staphylococcal infections with penicillin by intermittent sterilization. Lancet. ii, 497.

Cunliffe, A. C., \& Krickler, M. S. (1953). A cultural test for the presumptive serological identity of Proteus vulgaris. VIth Congr. int. Microbiol., Rome, 1, 400 .

Dienes, L. (1946). Reproductive processes in Proteus cultures. Proc. Soc. exp. Biol., N.Y. 63, 265.

Dienes, L. (1947). Further observations on the reproduction of bacilli from large bodies in Proteus cultures. Proc. Soc. exp. Biol., N.Y. 66, 97.

Hughes, W. H. $(1953 a)$. The origin of the $L$-form variants in anaerobic cultures of Bacterium coli. J. gen. Microbiol. 8, 307.

Hughes, W. H. (1953 b). Discussion in Adaptation in micro-organisms. Symp. Soc. gen. Microbiol. 3, 250.

Hughes, W. H. (1956). The structure and development of the induced long forms of bacteria in Bacterial Anatomy. Symp. Soc. gen. Microbiol. 6, 341.

KLIENEBERger-Nobel, E. (1947). Morphological appearances of various stages in B. proteus and coli. J. Hyg., Camb. 45, 410.

Kvittingen, J. (1949). Studies of the life-cycle of Proteus Hauser. Acta path. microbiol. scand. 26, 24.

Kvittingen, J. (1953). Studies of the life-cycle of Proteus Hauser. Acta path. microbiol. scand. 32, 170.

Lominski, I. \& Lendrum, A. C. (1947). The mechanism of swarming of Proteus. J. Path. Bact. 59, 688.

MoLTKE, $O$. (1927). Contributions to the characterisation and systematic classification of Bact. proteus vulgaris (Hauser). Copenhagen: Levin and Munksgaard.

Russ-Münzer, A. (Mayr-Harting) (1935). Das Schwärmphänomen bei Bacillus Proteus. Zbl. Bakt. (1. Orig.), 133, 214.

\section{EXPLANATION OF PLATE}

Fig. 1. Direct print of plate to show sectoring giving renewed growth on drying medium.

Fig. 2. Effect of unilateral drying of plate. Swarming only on medium where there is no concentration of agar. 
Journal of General Microbiology, Vol. 17, No. 1
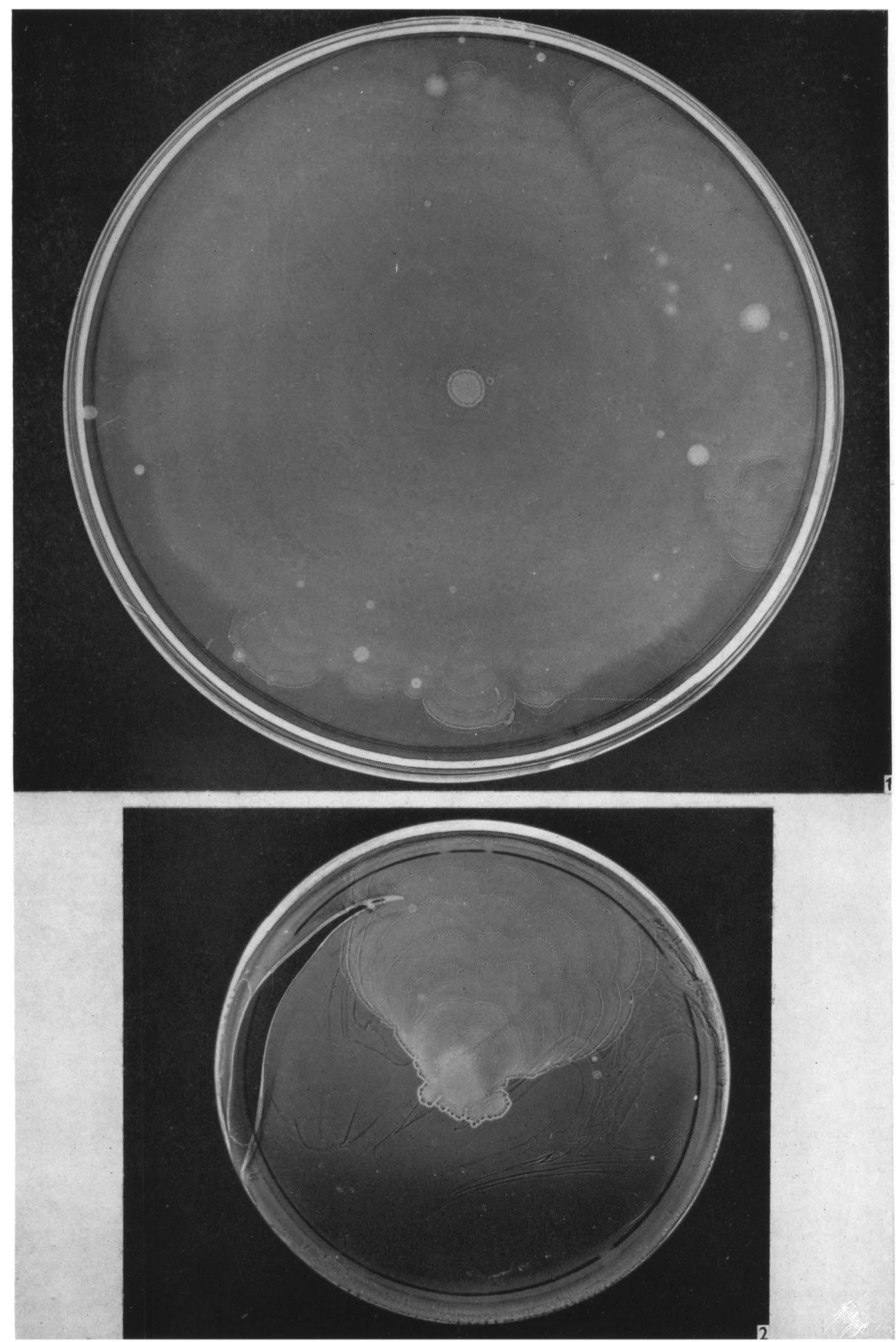

W. H. Hughes-The swarming of Proteus. Plate 1 